\title{
Downlink Packet Scheduling with Minimum Throughput Guarantee in TDD-OFDMA Cellular Network
}

\author{
Young Min Ki, Eun Sun Kim, Sung Il Woo, and Dong Ku Kim \\ Yonsei University, Dept. of Electrical and Electronic Engineering, \\ 134 Shinchon-Dong, Seodaemun-Gu, Seoul 120-749, Korea \\ \{mellow, esunkim, niceguy8189, dkkim\}@yonsei.ac.kr \\ http://mcl.yonsei.ac.kr
}

\begin{abstract}
This paper proposes a channel scheduler in downlink of IEEE 802.16e TDD-OFDMA cellular network which provides users the minimum throughput for best effort traffic. The proposed scheduler is further developed in the consideration of the case that network might become unfeasible, where extra weight functions are introduced into the proportional fair $(\mathrm{PF})$ scheduler so that the proposed extra weight function provides more resources to users who are easier to achieve the target throughput than those who cannot. It does not guarantee target throughput for all users but eventually increases the number of users being served with the guaranteed minimum throughput. It is investigated for the proposed schedulers and other conventional ones how large the throughput is and how many users are guaranteed the target throughput. Simulation results show that the proposed algorithms have 10 to $20 \%$ more users being served with the minimum guaranteed throughput. The trade-off between the throughput guarantee level and sector throughput is shown in the proposed schedulers.
\end{abstract}

\section{Introduction}

Emerging WLANs and future wireless mobile systems are expected to adopt a multi-carrier scheme (OFDM) consisting of hundreds of carriers [1]. For a broadband channel, fading over frequency as well as time should be handled more carefully in order to be able to enhance overall performance. There are two strategies applied to combat frequency variations: "frequency diversity" and "frequency selectivity" [2]. Frequency diversity, which employs the identical modulation and coding schemes over all subchannels, allows system performance not to be dominated by a few deeply faded subcarriers.

The frequency selective strategy exploits channel information over frequency to achieve adaptation gain, which is realized by the use of different link adaptations and channel schedulers in each subchannel. The studies of making the OFDMA more efficient in terms of frequency domain scheduling has been addressed in the number of papers [2-7]. However, it is still unclear what scheduling algorithm maximizes the sector throughput while still providing users with 
certain QoS such as minimum throughput or delay requirement. Such QoS guarantees would ensure that the network renders the service to all the users. In order to highlight this paradigm, the present paper evaluates the performance of scheduling algorithms under minimum user throughput guarantees in the IEEE 802.16e TDD-OFDMA [8-10] downlink network.

\section{Packet Scheduling for TDD-OFDMA Downlink}

\subsection{Downlink Scheduling for TDD-OFDMA}

The OFDMA is one of the time and frequency division multiple access techniques based on the OFDM and is currently used in the IEEE 802.16d [8] and 802.16e [9-10] standards. The fixed length OFDMA frame consists of a block of downlink (DL) OFDM symbols followed by another block of (UL) OFDM symbols. In the frequency domain, full RF bandwidth is divided into hundreds of subcarriers. Numbers of subcarriers are bunched into the subchannel, which can be handled as a minimal resource unit. The time division duplexing (TDD) does not provide either continuous downstream or continuous upstream. The channel feedback period is determined to be the same as OFDMA frame duration. Therefore, scheduling should be able to be performed at most every frame. Scheduling in OFDM/TDM allocates whole bands to only one user while OFDMA can assign different users over different subchannels. Fig. 1 shows an example of channel scheduling that happens in TDD-OFDMA where single OFDMA scheduler is capable to assign different users to different subchannels [2][5].

\subsection{Conventional Scheduling Methods}

1) Maximum $C / I(\operatorname{Max} C / I)$ : One simple method is to serve the user terminal (UT) of index $i_{n}^{*}$ at the $n$-th subchannel for every scheduling instance $t$ with respect to:

$$
i_{n}^{*}=\arg \max _{i} R_{i, n}(t)
$$

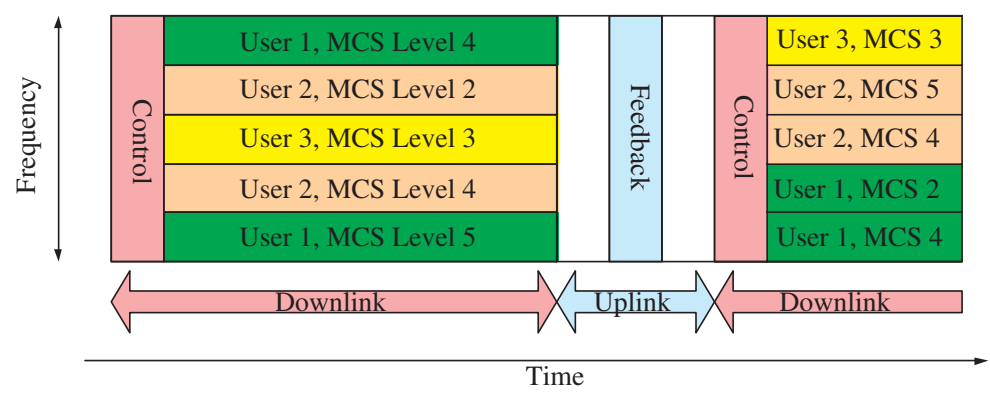

Fig. 1. Priority-based channel scheduling example in TDD-OFDMA downlink 
where $R_{i, n}(t)$ denotes the instantaneous supportable data rate of the $n$-th subchannel of the user $i$. This serving principle has obvious benefits in terms of system throughput. However, it does not take into consideration of throughput fairness of users, leaving those users of poor average radio conditions served less frequently.

2) Proportional Fair $(P F)$ : To remedy the fairness problem, the proportional fairness (PF) scheduling algorithm was proposed [11-12]. According to the PF scheduling algorithm, the selected UT of index $i_{n}^{*}$ should be denoted such that:

$$
i_{n}^{*}=\arg \max _{i} \frac{\left(R_{i, n}(t)\right)^{\alpha}}{\left(T_{i, n}(t)\right)^{\beta}},
$$

where $T_{i, n}(t)$ denotes the average throughput of the $n$-th subchannel of UT $i$. $\alpha$ and $\beta$ are indices used to control the scheduling fairness, which are normally set to $\alpha=\beta=1$. The PF algorithm intends to serve those users seeing very favorable instantaneous radio channel conditions relative to their average ones, thus taking advantage of the temporal variations of the fast fading channel.

3) Fast Fair Throughput (FFTH): In [13], the fast fair throughput (FFTH) scheduler was suggested that the selected UT of index $i_{n}^{*}$ should be defined that:

$$
i_{n}^{*}=\arg \max _{i} \frac{R_{i, n}(t)}{T_{i, n}(t)} \cdot\left[\frac{\max _{j}\left(\overline{R_{j, n}(t)}\right)}{\overline{R_{i, n}(t)}}\right],
$$

where $R_{i, n}(t) / T_{i, n}(t)$ is a well-known PF factor and $\overline{R_{i, n}(t)}$ is the average supportable data rate of the $n$-th subchannel of the user $i$. The expression $\max _{j}\left(\overline{R_{j, n}(t)}\right)$ is a constant that indicates the maximum average supportable data rate from all the users. Note that the $\overline{R_{i, n}(t)}$ term in the denominator is to compensate the priority of less favorable users and distribute evenly the cell throughput to all users.

4) Fair Throughput (FTH): For throughput fairness in wireless, a simplemethod is considered to serve the user terminal (UT) of index $i_{n}^{*}$ at the $n$-th subchannel for every scheduling instance $t$ with respect to:

$$
i_{n}^{*}=\arg \max _{i} \frac{1}{T_{i, n}(t)},
$$

where $T_{i, n}(t)$ denotes the average throughput of the $n$-th subchannel of UT $i$. The FTH scheduling is considered as a slow scheduling due to the fact that it does not require any instantaneous information of the channel quality [13].

\section{Schedulers for Minimum Throughput Guarantee}

\subsection{User Throughput Outage}

If no constraint were imposed on users' QoS, the Max C/I scheduler would provide maximum system throughput. The fair scheduling algorithms such as PF, 
FFTH dent and FTH represent the trade-off between throughput and fairness, but cannot provide any QoS guarantees such as minimal throughput. For highly loaded conditions, users having poor average radio channel conditions are allocated very little time resources, starved for throughput, and will ultimately be dropped out of the network without satisfying their requirements [5][13]. A set of user throughput is said to be feasible if the sum of all the user throughputs is lower or equal to the air interface capacity. If the network is feasible, there are certain schedulers that can guarantee all the requirements. In order to be able to guarantee the user throughput requirements, the network should be able to provide a minimum throughput to all users with a certain outage levels allowed in the network. The throughput outage probability of the user $i$ is defined as the probability that user throughput cannot satisfy the required target throughput which is expressed as follows:

$$
\operatorname{Pr}_{i}\left(T_{i}<T_{i, r e q}\right) \leq \delta_{i}
$$

where $T_{i}$ is the average throughput of user $i$ and $T_{i, r e q}$ is the required minimum throughput of the user $i$ that can be determined by user QoS class. $\delta_{i}$ is the required target throughput outage probability of user $i$.

\subsection{Extra Weight Method for Minimum Throughput Guarantee}

In [14], various modifications of the PF algorithm were proposed to guarantee the delay requirements. In this paper, the modification of PF algorithm of [14] is introduced for the minimum user throughput guarantees. The selected UT of $i_{n}^{*}$ of our modified algorithm is denoted such that:

$$
i_{n}^{*}=\arg \max _{i}\left\{\begin{array}{ll}
\frac{\left(R_{i, n}(t)\right)^{\alpha}}{\left(T_{i, n}(t)\right)^{\beta}} \cdot C_{i, n}, & T_{i}<T_{i, r e q} \text { or } \operatorname{Pr}_{i}\left(T_{i}<T_{i, r e q}\right)>\delta_{i} \\
\frac{\left(R_{i, n}(t)\right)^{\alpha}}{\left(T_{i, n}(t)\right)^{\beta}}, & T_{i} \geq T_{i, r e q} \text { and } \operatorname{Pr}_{i}\left(T_{i}<T_{i, r e q}\right) \leq \delta_{i}
\end{array},\right.
$$

where $\left(R_{i, n}(t)\right)^{\alpha} /\left(T_{i, n}(t)\right)^{\beta}$ is the well-known PF factor and $C_{i, n}$ is extra weight of the $n$-th subchannel of user $i . \operatorname{Pr}_{i}\left(T_{i}<T_{i, r e q}\right)$ is the throughput outage probability and $P t_{t}$ is target outage of user $i$. Only needy users are given extra weight $C_{i, n}$ once either user throughput turns out to be unable to guarantee the minimum user throughput or the throughput outage exceeds a certain target level. When there is no throughput violations or $C_{i, n}=1$, this scheduling algorithms becomes to be equivalent to a conventional $\mathrm{PF}$ algorithm. The extra weight $C_{i, n}$ is given as (7):

$$
C_{i, n}=\frac{\max _{j}\left(\overline{R_{j, n}(t)}\right)}{\overline{R_{i, n}(t)}},
$$

which is the same form as the weighting function of FFTH of (3). $C_{i, n}$, which is the ratio of the maximum supportable data rate of the system to average supportable data rate of needy user, would allocate more resources to those users who have inferior throughput outage probability. 


\subsection{Minimum User Throughput in Unfeasible Networks}

Since the supportable data rates of users are determined by channel condition in the rate controlled scheduling, the users in the cell edge, who are likely to have lower SINR than one which is required for the transmission of minimum data rate, cannot be served so much as to meet the minimum user throughput. In this case, the increase in priority of the users in this unfeasible area cannot guarantee the minimum user throughput but only decrease system throughput. Since $C_{i, n}$ of (7) would have given too much extra priority to poor channel condition users in unfeasible conditions, it is modified as follows:

$$
C_{i, n}=\left[\frac{\max _{j}\left(\overline{R_{j, n}(t)}\right)}{\overline{R_{i, n}(t)}}\right]^{\min \left(1.0, T_{i} / T_{i, r e q}\right)},
$$

where $T_{i} / T_{i, r e q}$ is the normalized throughput. If the user throughput is close to the required target throughput, the exponent $\min \left(1.0, T_{i} / T_{i, r e q}\right)$ becomes to be close to 1.0. If the user throughput becomes smaller, the exponent goes to 0.0 . Therefore, the extra priority of (8) cannot guarantee all of minimum user target throughputs. The extra priority of (8) is to provide those users who are easier to achieve the target throughput with more service than those who cannot.

\section{Simulation Results}

\subsection{Simulation Environments}

Table 1 shows the IEEE 802.16e based OFDMA parameters [8-10] and the link adaptation table (MCS table) is set to the parameters displayed in Table 2 [15-16]. The MCS level is reported to mobiles according to SINR sensitivity thresholds and one frame delay is assumed in MCS feedback. It is assumed that there are 19 cells with 3 sectors of same frequency allocation (FA) and cell radius is $1 \mathrm{~km}$. User terminals are distributed in the center sector and their locations are generated more than 1,000 times. Only best-effort traffic with full-buffering is considered. The channel $\mathrm{C} / \mathrm{I}$ of the $n$-th subchannel of user $i$ can be expressed as follows:

$$
(C / I)_{i, n}=\sum_{j=1}^{J}\left\|\gamma_{j, n}\right\|^{2} \cdot\left(G_{i}^{-1}+\sum_{k=1}^{K}\left\|\psi_{k, n}\right\|^{2}\right)^{-1},
$$

where $G_{i}$ is the average geometry which is determined by path loss and shadowing and shown as

$$
G_{i}=\frac{I_{o r}}{I_{o c}+N_{0} W}=\frac{1}{I_{o c} / I_{o r}+1 /\left(I_{o r} / N_{0} W\right)},
$$

where $I_{o r}$ is the received serving-cell pilot strength, $I_{o c}$ is the sum of the received other-cell pilot strength, and $N_{0} W$ is the thermal noise power. The expression $\left\{\gamma_{j}\right\}$ represents the multi-path component within the guard interval 
Table 1. OFDMA parameters

\begin{tabular}{cc}
\hline Parameters & Value \\
\hline Carrier Frequency & $2.3 \mathrm{GHz}$ \\
Channel Bandwidth & $10 \mathrm{MHz}$ \\
Number of used subcarriers & 1,702 of 2,048 \\
Number of traffic subcarriers & 1,536 \\
Subcarrier spacing & $5.57617 \mathrm{kHz}$ \\
Number of subchannels & 32 \\
Number of subcarriers & 48 \\
Frame length & $5.0 \mathrm{msec}$ \\
Number of symbols per frame & 26 \\
Number of DL / UL symbols & $18 / 8$ \\
Sum of RTG and TTG & $45.885 \mu \mathrm{sec}$ \\
OFDMA symbol time & $190.543 \mu \mathrm{sec}$ \\
Cyclic prefix & $1 / 16$ \\
\hline
\end{tabular}

Table 2. Modulation and coding (MCS) table

\begin{tabular}{cccc}
\hline Modulation & Code rate & Sensitivity threshold S/N & PHY bit/sec/Hz \\
\hline QPSK & $1 / 12$ & $-3.0 \mathrm{~dB}$ & 0.135 \\
QPSK & $1 / 8$ & $-1.3 \mathrm{~dB}$ & 0.202 \\
QPSK & $1 / 4$ & $1.4 \mathrm{~dB}$ & 0.404 \\
QPSK & $1 / 2$ & $6.6 \mathrm{~dB}$ & 0.807 \\
QPSK & $3 / 4$ & $8.5 \mathrm{~dB}$ & 1.211 \\
16QAM & $1 / 2$ & $10.5 \mathrm{~dB}$ & 1.613 \\
64QAM & $2 / 3$ & $15.3 \mathrm{~dB}$ & 3.227 \\
64QAM & $3 / 4$ & $20.8 \mathrm{~dB}$ & 3.63 \\
\hline
\end{tabular}

and $\left\{\psi_{k}\right\}$ is multi-path component which exceeds the guard interval. In simulations, $\sum_{j=1}^{J}\left\|\gamma_{j, n}\right\|^{2}$ is assumed to be Rayleigh fading and $\left\{\psi_{k}\right\}$ is ignored since the cyclic prefix is assumed to be sufficiently longer than the overall delay spread [12]. The path loss model is assumed to be a vehicular model $129.427+37.6 * \log _{10}\left(d_{k m}\right)$. The standard deviation of Log-normal shadowing is assumed to be $10 \mathrm{~dB}$. Short-term channel gains are assumed to be Rayleigh fading with a Doppler frequency of $6.4 \mathrm{~Hz}(3 \mathrm{~km} / \mathrm{Hr})$. The BS transmit power is set to $20 \mathrm{~W}(43 \mathrm{dBm})$. Thermal noise density is assumed to be $-174 \mathrm{dBm} / \mathrm{Hz}$ and $\max \mathrm{C} / \mathrm{I}$ limit is set to $30 \mathrm{~dB}$.

\subsection{Sector Throughput Performance}

Fig. 2 represents the users' average geometry distribution. It is shown that around $45 \%$ of samples has average geometry inferior to the lowest level which is $-3.0 \mathrm{~dB}$ as shown in Table 2. In this paper, the interference management tech- 


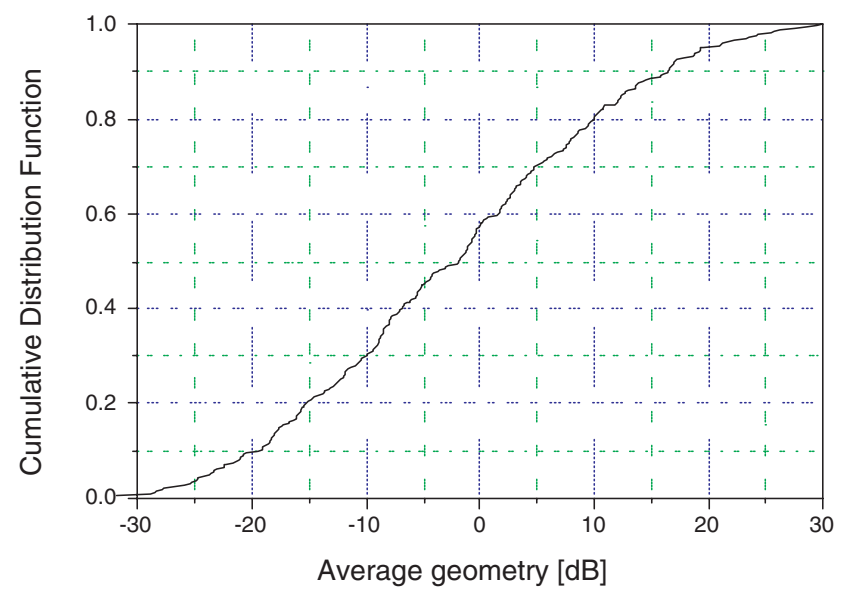

Fig. 2. Users' average geometry distribution

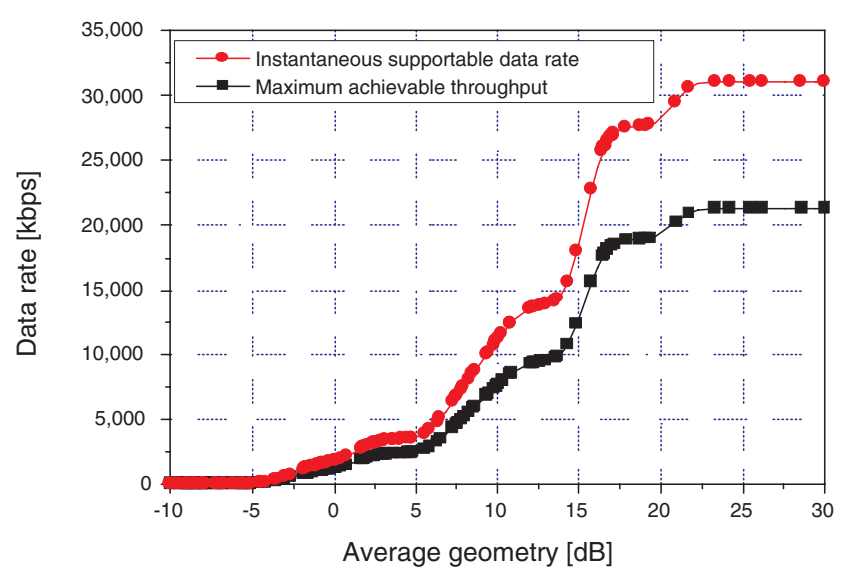

Fig. 3. Instantaneous supportable data rate and maximum achievable throughput according to average geometry

niques such as frequency hopping (FH) or handoff subchannel [9-10] are not considered and system could have trouble of being unfeasible due to those users in the poor channel conditions. Fig. 3 shows the instantaneous supportable data rate and maximum achievable throughput according to average geometry. Since TDD-OFDMA downlink transmits on DL subframe which uses 18 symbols out of 26 symbols, the maximum achievable throughput is around $70 \%$ of instantaneous supportable data rate in parameters of Table 1. Fig. 4 represents sector throughput performance of different scheduling methods for the various number of simulated users. Max C/I scheduler shows the largest multi user diversity gains as number of users increases. FTH and FFTH schedulers attempt to allo- 


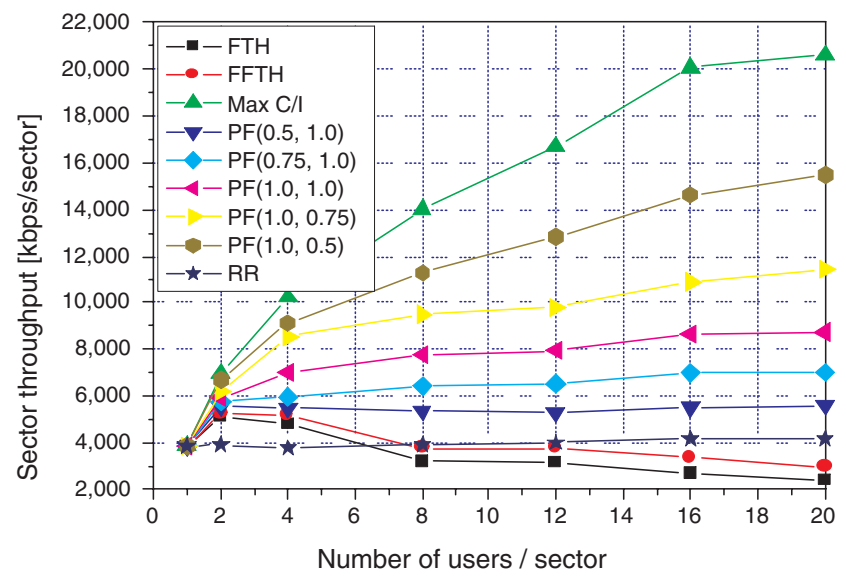

Fig. 4. Sector throughput results of different scheduling methods according to number of simulated users (fairness indices are presented as $\mathrm{PF}(\alpha, \beta)$ in the graph)

cate more resources to those lower throughput users, so provide smaller sector throughput as number of users increase. PF scheduler gives more emphasis on channel factor $(\alpha>\beta)$, so more multi user diversity gains are achieved.

\subsection{Minimum User Throughput Guarantee Performance}

In order to evaluate the throughput guarantee performance of different schedulers, three minimum target throughputs are considered: 64, 128, and $384 \mathrm{kbps}$. Target throughput outage is set to $5 \%$ for all three cases. Two versions of proposed algorithm of (6) are evaluated: the proposed Algorithm 1 has extra function of (7) and Algorithm 2 adopts extra function of (8). Fairness indices of proposed algorithms are set to $\alpha=\beta=1$. It was evaluated how many users in average are guaranteed their target throughput and how large is their average throughput. Table 3 shows the minimum throughput guarantee results when the number of simulated users is set to 16 . For $64 \mathrm{kbps}$ guarantee, all scheduling algorithms except max $\mathrm{C} / \mathrm{I}$ provide similar number of guaranteed users but PF and proposed algorithms provide around 2.5 to 3 times more throughput than FTH, FFTH and RR. In this case, the throughput requirement is not tight and all schedulers show good performance. For $128 \mathrm{kbps}$ case, the proposed Algorithm 2 shows the most guaranteed users but show around $30 \%$ throughput loss than PF, which means that the proposed algorithm allocates more resources to throughput violated users than PF. In order to test our algorithms in high loaded unfeasible network, we increase target throughput up to $384 \mathrm{kbps}$. The FTH, FFTH and Algorithm 1 turns out to give too much resources to those users in poor channel conditions, so it could not guarantee the minimum user throughput and only decrease system throughput. However, the proposed Algorithm 2 guarantees around $25 \%$ more users while decreasing system throughput. 
Table 3. Minimum user throughput guarantees of 64 , 128, and $384 \mathrm{kbps}$ throughput with $5 \%$ target outage when the number of simulated users is 16

\begin{tabular}{|c|c|c|c|c|}
\hline $\begin{array}{l}\text { Target } \\
\text { outage }\end{array}$ & $\begin{array}{l}\text { Scheduling } \\
\text { Algorithm }\end{array}$ & $\begin{array}{c}\text { Average } \\
\text { number of } \\
\text { guaranteed } \\
\text { users }\end{array}$ & $\begin{array}{c}\text { Average } \\
\text { throughput of } \\
\text { guaranteed } \\
\text { users }[\mathrm{kbps}]\end{array}$ & $\begin{array}{c}\text { Total } \\
\text { throughput of } \\
\text { guaranteed } \\
\text { users [kbps] }\end{array}$ \\
\hline \multirow{7}{*}{$\begin{array}{c}64 \mathrm{kbps} \\
(5 \%)\end{array}$} & $\operatorname{Max} \mathrm{C} / \mathrm{I}$ & 1.49 & $13,456.39$ & $20,050.02$ \\
\hline & $\mathrm{RR}$ & 8.05 & 495.10 & $3,985.56$ \\
\hline & FTH & 9.53 & 281.53 & $2,682.98$ \\
\hline & FFTH & 9.43 & 355.55 & $3,352.84$ \\
\hline & $\mathrm{PF}$ & 9.21 & 935.40 & $8,615.30$ \\
\hline & Algorithm 1 & 9.55 & 895.34 & $8,550.50$ \\
\hline & Algorithm 2 & 9.67 & 828.21 & $8,008.79$ \\
\hline \multirow{7}{*}{$\begin{array}{c}128 \mathrm{kbps} \\
(5 \%)\end{array}$} & $\operatorname{Max} \mathrm{C} / \mathrm{I}$ & 1.43 & $13,996.38$ & $20,014.82$ \\
\hline & $\mathrm{RR}$ & 6.20 & 638.48 & $3,958.58$ \\
\hline & FTH & 9.25 & 286.21 & $2,647.44$ \\
\hline & FFTH & 8.78 & 373.10 & $3,275.82$ \\
\hline & $\mathrm{PF}$ & 8.11 & $1,048.19$ & $8,500.82$ \\
\hline & Algorithm 1 & 8.84 & 823.86 & $7,282.92$ \\
\hline & Algorithm 2 & 9.29 & 736.18 & $6,389.11$ \\
\hline \multirow{7}{*}{$\begin{array}{c}384 \mathrm{kbps} \\
(5 \%)\end{array}$} & Max C/I & 1.27 & $15,746.70$ & $19,998.31$ \\
\hline & $\mathrm{RR}$ & 4.10 & 928.94 & $3,808.65$ \\
\hline & FTH & 0.83 & 565.76 & 469.58 \\
\hline & FFTH & 2.65 & 556.68 & $1,475.21$ \\
\hline & $\mathrm{PF}$ & 5.34 & $1,465.90$ & $7,827.91$ \\
\hline & Algorithm 1 & 2.64 & 644.41 & $1,701.24$ \\
\hline & Algorithm 2 & 6.74 & 467.63 & $3,151.83$ \\
\hline
\end{tabular}

\subsection{Geographical Fairness Performance}

A Ring is defined as the area occupied by a 100 meter unit. For example, the $r$-th Ring is the area which falls into between the $(r-1)$ hundreds meters and the $r$ hundred meters from the BS. "Average user throughput of the $r$-th Ring" represents a user average rate in the $r$-th Ring and is determined as:

$$
R_{\text {user }}(r)=\frac{R_{\text {ring }}(r)}{\text { Number of User in } r \text {-th Ring }},
$$

where $R_{\text {ring }}(r)$ is "Ring throughput" of the $r$-th Ring and is defined as:

$$
R_{\text {ring }}(r)=\sum_{i \in r \text {-th Ring }} T_{i}=R_{\text {user }}(r) \cdot N_{\text {user }}(r),
$$

where $T_{i}$ denotes average throughput of the $i$-th user and $N_{u s e r}(r)$ denotes number of users in of the $r$-th Ring. Fig. 5 represents average user throughput per Ring in PF and proposed algorithms when the number of simulated users is 16 . 


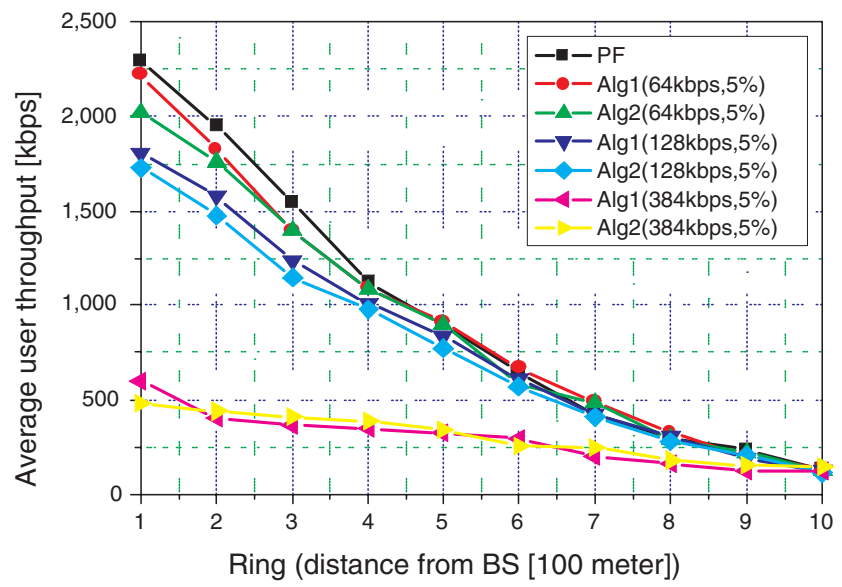

Fig. 5. Average user throughput per Ring in PF and proposed scheduling algorithms when the number of simulated users is 16

In 64 and 128 kbps target throughput guarantee cases, Algorithm 1 shows better throughput performance than Algorithm 2, but Algorithm 2 provides more guaranteed users. However, for $384 \mathrm{kbps}$ case, Algorithm 1 outperforms to Algorithm 2 only in the first Ring. It is also shown that the user throughput becomes more geographically fair for higher target throughput, resulting in total throughput decrease.

\section{Concluding Remarks}

The total throughput and minimum throughput guarantee performance of best effort service were evaluated for downlink of an IEEE 802.16e TDD-OFDMA cellular network. The conventional schedulers without minimum throughput guarantee shows trade-off between throughput and fairness. To be able to guarantee the minimum user target throughput, the extra weight functions were introduced into the proportional fair (PF) scheduler. The proposed extra weight algorithms provide users who are easier to achieve the target throughput with more service than those who cannot, among those users not satisfying the target throughput due to the poor channel conditions that might also make system become at the risk of being unfeasible. The proposed algorithm does not guarantee target throughput for all users but eventually increase the number of the guaranteed users. Simulation results show that the proposed algorithms represent 10 to $20 \%$ more users who can be served with the minimum guaranteed throughput. 


\section{Acknowledgments}

This work was supported by the Korea Research Foundation Grant (KRF-2004013-D00060). Also, this work was supported by grant No. (R01-2002-000-00531-

0) from the Basic Research Program of the Korea Science \& Engineering Foundation.

\section{References}

1. Shakkottai S., Rappaport T. S.: Cross-Layer Design for Wireless Networks. Communications Magazine, Vol. 41. IEEE. (2003) 74-80

2. Classon B., Sartori P., Nangia V., Zhuang X., Baum K.: Multi-dimensional Adaptation and Multi-user Scheduling Techniques for Wireless OFDM Systems. IEEE International Conference on Communications (ICC '03), (2003)

3. Anchun W., Liang X., Shidong Z., Xibin X., Yan Y.: Dynamic Resource Management in the Forth Generation Wireless Systems. International Conference on Communication Technology (ICCT2003), (2003)

4. Xiao L., Wang A., Zhou S., Yao Y.: A Dynamic Resource Scheduling Algorithm for OFDM System. The 9th Asia-Pacific Conference on Communications, 2003 (APCC 2003)

5. Ki Y. M., Kim E. S., Kim D. K.: Downlink Scheduling and Resource Management for Best Effort Service in TDD-OFDMA Cellular Networks. Lecture Notes in Computer Science, Vol. 3260. (2004) 315-329

6. Das S., Viswanathan H.: Dynamic Power and Sub-carrier Assignment in a Multiuser OFDM System. IEEE 60th Vehicular Technology Conference (VTC 2004)

7. Kim K., Kim H., Han Y.: Subcarrier and Power Allocation in OFDMA Systmes. IEEE 60th Vehicular Technology Conference (VTC 2004)

8. http://www.ieee802.org/16/tgd/

9. http://www.ieee802.org/16/tge/

10. IEEE P802.16e/D3, Draft Amendment to IEEE Standard for Local and Metropolitan Area Networks, Part 16: Air Interface for Fixed Broadband Wireless Access Systems - Amendment for Physical and Medium Access Control Layers for Combined Fixed and Mobile Operation in Licensed Bands, (2004)

11. Jalali A., Padovani R., Pankaj P.: Data throughput of CDMA-HDR a high efficiency-high data rate personal communication wireless system. The 51st IEEE Vehicular Technology Conference, 2000 (VTC 2000-Spring)

12. 3GPP R1-030042, Update of OFDM SI simulation methodology. (2003)

13. Ameigeiras P., Wigard J., Mogensen P.: Performance of Packet Scheduling Methods with Different Degree of Fairness in HSDPA. IEEE 60th Vehicular Technology Conference (VTC 2004)

14. Barriac G., Holtzman J.: Introducing Delay Sensitivity into the Proportional Fair Algorithm for CDMA Downlink Scheduling. IEEE Seventh International Symposium on Spread Spectrum Techniques and Applications (2002)

15. IEEE C802.16e-03/22r1, Convergence simulations for OFDMA PHY mode, (2003)

16. IEEE C802.16d-03/78r1, Coverage/Capacity simulation for OFDMA PHY in with ITU-T channel model, (2003) 